\title{
Individualized, Low-Cost And Accessible Pulmonary Rehabilitation Program Based On Functional Clinical Tests For Individuals With Copd- A Study Protocol Of A Randomized Controlled Trial
}

Marcela Maria Carvalho da SILVA ( $\sim$ marcelacarvalhofisioterapia@yahoo.com.br )

Federal University of São Carlos https://orcid.org/0000-0003-0723-403X

JULIANO FERREIRA ARCURI

Federal University of Sao Carlos: Universidade Federal de Sao Carlos

VALERIA AMORIM PIRES DI LORENZO

Federal University of Sao Carlos: Universidade Federal de Sao Carlos

Study protocol

Keywords: rehabilitation, exercise therapy, COPD, Physical Functional Performance

Posted Date: January 18th, 2021

DOl: https://doi.org/10.21203/rs.3.rs-146768/v1

License: (c) (i) This work is licensed under a Creative Commons Attribution 4.0 International License.

Read Full License 


\section{Abstract}

Backgroud:

Patients with Chronic Obstructive Pulmonary Disease (COPD) present pulmonary and extrapulmonary impairments. In order to mitigate these impairments, pulmonary rehabilitation programs (PRP) it is an important strategy but the access to PRP in specialized center is limited and the studied of low-cost home rehabilitation programs had non-individualized prescription, which might have led to insignificant positive effects. So, it is important to develop new low-cost protocols that involves individualized prescription, and the influence of physiotherapist supervision. The aim of this study was to describe an accessible, lowcost and individualized pulmonary rehabilitation protocol and compare its results when performing it with or without a weekly physiotherapist-supervised session on patients with COPD.

Methods: This is a descriptive protocol of a clinical trial with parallel equivalent groups, conducted at the Spirometry and Respiratory Physical Therapy Laboratory of the Federal University of São Carlos (UFSCar). The trial was registered at Brazilian Clinical Trials Registry (ReBec) URL:

http://www.ensaiosclinicos.gov.br/rg/RBR-533hht/ with Register Number RBR-533hht. The sample size was 40 patients and was calculated using the results of a pilot study.

Discussion- potencial impact and significance of the study: It is expected that the low cost and new supervised rehabilitation program complemented with home exercises will present positive results especially on exercise capacity, which will make available a more accessible and effective PRP for patients with COPD.

Trial registration: The name of the registry was Responsibility of functional tests on supervised rehabilitation program and education program in patients with chronic obstructive pulmonary disease,

with registration number. RBR-533hht. The date of registration and start data was September 20, 2018.

\section{Backgroud}

Patients with Chronic Obstructive Pulmonary Disease (COPD) present pulmonary impairments, such as dyspnea, pulmonary hyperinflation, gas exchange deficit and decreased respiratory-muscle endurance and strength. Moreover, extra pulmonary manifestations on skeletal muscles, cognitive, nutritional and cardiovascular functions which lead to reduced physical-activity levels, exercise capacity, functionality and quality of life, and ultimately increase morbidity and mortality $(1,2) \cdot(3)$.

In order to mitigate these impairments, pulmonary rehabilitation programs (PRP) aim to increase autonomy on physical, social and emotional performance, positively influencing symptoms, disease progression and increasing exercise tolerance. Hence, quality of life, exercise capacity and psychological condition are improved and morbidity, health costs and mortality are reduced $(4,5)$. 
In spite of PRP benefits, less than 10\% of patients diagnosed with COPD have access to a specialized service and several causes are associated with this, as lack of rehabilitation centers specially in country side and rural areas, dislocation difficulties due to poor physical condition and mobility limitations and family dependency. Furthermore, there is a high cost in the construction and maintenance of these centers (6), which decreases accessibility and feasibility. Therefore, alternative strategies to conduct a PRP have been investigated.

Different technological levels are among the possible strategies to address this matter. High technological aids, such as telerehabilitation are characterized by activities performed by patients at home with professional supervision by communication technologies such as computers, internet and remote control and monitoring. Thus, there is no patient's dislocation need, however, the high cost prevents its feasibility to low-income populations (7).

Low level technologies (with low cost) are also described in literature and they include educational charts, domiciliary exercise instructions and functional physical activities(8). The advantage of this method is the feasibility, since they may be conducted in primary care, low-technology rehabilitation centers or patient's home. Previous studies verified the effect of a low-cost pulmonary rehabilitation executed in patients' home and supervised by a physical therapist, they concluded that domiciliary PRP are a valid alternative (9)'(6)'(10). Nevertheless, in most studies, exercise prescription was not individualized, and greater results might be found since the empirical protocol may underestimate patient's capacity, or overestimate, leading to muscle soreness, fatigue, thus patients may give up treatment. Hence, the lack of individualized prescription is not congruent withthe marked heterogeneity of intervention and disease phenotypes(6)'(11).

Furthermore, although previous studies(10),(6) did not find differences between a low-cost home and center-based rehabilitation, however, both groups did not obtained minimal clinical difference in Borg scale (Holland et al, 201) and walked distance on six-minute walk test (6MWT). Still, the incremental of exercise prescription was not the same in both groups of rehabilitation and it leads a lack of interpretation on the results, being influenced not only by environmental executed, but also in prescription of exercise on PRP.

Therefore, considering that the access to PRP in specialized center is limited and the studied of low-cost home rehabilitation programs had non-individualized prescription, which might have led to insignificant positive effects, it is important to develop new low-cost protocols that involves individualized prescription, and the influence of physiotherapist supervision.

So, the aim of this study was to describe an accessible, low-cost and individualized pulmonary rehabilitation protocol and compare its results when performing it with or without a weekly physiotherapist-supervised session on patients with COPD.

\section{Methods}




\section{Trial design and study settings}

This is a descriptive protocol of a clinical trial with parallel equivalent groups, single blinded conducted at the Spirometry and Respiratory Physical Therapy Laboratory of the Federal University of São Carlos (UFSCar).

The study protocol was developed according to Standard Protocol Items: Recommendations for Interventional Trials (SPIRIT) 2013 Checklist guidelines (appendix 1 and Fig. 1) and the trial was registered at URL: http://www.ensaiosclinicos.gov.br/rg/RBR-533hht/ with Register Number RBR-533hht. So, the trials status is: the date of registration and start data was September 20,2018 , the actual status is ongoing and the end prevision is December, 2020.

The protocol was approved by Research Ethics Committee of the Federal University of São Carlos (UFSCar) number 4.348.948, CAAE: 85901318.0.0000.5504 and all volunteers will be responsible by signed

an informed consent form.

The personal information about potential and enrolled participants will be confidencial. This information will be not shared, however it will be consulted by researches of this study. Finally, the information will be used for scientific purposes, always safeguardion your provacity before, during, and after the trial.

\section{Availability of data and materials}

The evaluation and the intervention will be executed at the Spirometry and Respiratory Physical Therapy Laboratory of the UFSCar. The data will be availability in the center and the researchers will be responsible for the assessment, reassessment, and supervision of the intervention.

\section{Eligibility criteria inclusion and exclusion criteria}

The inclusion criteria are (i) age between the range 50-85 years, (ii) diagnosis of COPD, (iii) absence of exacerbation and stability of drug treatment in the last four weeks, (iv) absence of PRP in the last six months; (v) Modified Medical Research Council (mMRC) greater than or equal to two, (vi) low exercise capacity verified by the results of six-minute step test and / or six-minute walk test (lower than $80 \%$ of predicted value); lack of cognitive impairment with language deficiency that impairs to understanding the verbal instructions.

The exclusion criteria are conditions that impair the execution of proposed tests (12), such as unstable angina and myocardial infarction on previous months, arrhythmias, unstable blood pressure and bodymass index greater than $35 \mathrm{~kg} / \mathrm{m} 2$; malignant cancer in treatment; orthopedic, rheumatic, neuromuscular and visual problems that make it impossible to walk and / or go up and down stairs.

\section{Recruitment strategies}


Patients undergoing medical treatment at public outpatient pulmonology services in São Carlos will be invited to participate in the research according to eligibility criteria. After acceptance, all participants will sign a consent form and will undergo a spirometry(13) (on the first day of evaluation), clinical and physical evaluation (on the second and the third day of evaluation) at the Spirometry and Respiratory Physiotherapy laboratory of UFSCAR.

\section{Outcomes}

Patient's assessment will be conducted in a standard sequence, which is presented in Fig. 2 is assessment will be conducted in two visits, with at least seven days between them. The procedure will be also executed before and after PRP to verify the effects of the program.

The baseline characteristics of this study will be information about the patients' anthropometric data, schooling level, presence of continuous use of oxygen therapy, ongoing pulmonary rehabilitation, active smoking, comorbidities (Charlson Comorbidity Index) and pulmonary function assessed by spirometry (13)

The primary outcome of this study will be the number of steps in the six-minute step test (6MST), which will also be used to guide aerobic training prescription. A single trial of a 6MST will be performed using the standardized technique (14)'(15) and the number of steps will be registered and compared with normative values(16).

The secondary outcomes will be:

1. Six minute walk test. Two $6 \mathrm{MWTs}$ will be performed using the standardized technique(12), for statistical analysis we will considering the greatest distance walked between the two tests. The distance will be compared with normative values(17).

2. (14)One minute sit to stand test. $1 \mathrm{MST}$ will be performed using the standardized technique (18) and the number of sit to stand will registered and compared with normative value (19)

3. Modified Medical Research Council (mMRC): Is as a questionnaire graduated 0 to 4 scores and the highter the score the worst the dispneia(20).

4. Ten repetition maximum test. will be performed on diagonally of movement of domiant upper limb with a incremental free weight(21).

5. Quality of life: The Saint George Respiratory Questionnaire (SQRQ)and COPD Assessment Test (CAT) (22) will be executed by the same evaluator with interview format.

6. Physical Activity daily living (PADL): actígraph activPAL3TM (PAL Technologies Ltd., Glasgow, Reino Unido) will be fixed (Tegaderm-3M) in the middle of right thigh (23) during seven days. The main variables will be: average daily sitting time and standing time (seven days), daily number of steps and intensity of exercise.(24)

\section{Randomization}


Patients will be randomized in two groups: instruction group (IG) and supervised group (SG). The allocation sequence generation will be done by computer-generated random numbers (online version) and allocation concealment will be done by sealed brown envelope, the sequence will be generated and kept by researcher who will not be involved in the recruitment, evaluation or training of patients, who will be reached by the researcher responsible for the intervention by a telephone call. The assessor will be blind to group allocation.

The protocol will be executed by a single training physiotherapist who is not involved on evaluation or randomization process.

\section{Intervention}

The intervention will last eight weeks and will be started one week after the assessment is finished. The intervention has two distinct moments: personalized instructions and exercise prescription. The exercise prescription is based on the performance in functional physical tests and it is composed by warm up, aerobic exercise, muscle strengthening and stretching. The exercise load was progressive according to individual tolerance and performance on clinical tests, according to protocol created by our research group, based in previous studies(6)'(4).

\section{Personalized instructions (booklet)}

On the first day of meeting with the physiotherapist responsible for the rehabilitation, all patients in both groups will participate in one educational session organized in a booklet, composed by instructions regarding COPD physiopathology, pulmonary and extrapulmonary manifestations, multidisciplinary treatment, involving smoking cessation, correct medication administration, vaccination, symptom control, and benefits of practicing physical activity (appendix 2). Furthermore, patients will be instructed about signs to interrupt exercise, exercise safety and symptom control (appendix 2) and environmental changes to facilitate exercise practice.

\section{Warm up}

This phase is composed by mild intensity physical activity (Ex. Light walk) during three to five minutes (see progression in appendix 3).

Aaerobic exercise: This phase includes more vigorous walking, sit to stand movements and step up and down. The weekly progression of these exercises (appendix 3 ) will consider the following variables for all patients: (i) the results from the maximum tolerance in the assessed functional physical tests (6MWT, 6MST and 1MSST): Initially, a suggested exercise intensity/volume of $80 \%$ of the verified performance in the assessed functional physical tests will guide exercise training, the weekly progression will achieve up to $100 \%$ of that performance (see progression in appendix 3); (ii) symptom-limited: during the execution of the exercise at the suggested intensity/volume, patients shall maintain Borg scale in a range of 3 to 5 points (moderate to intense effort perception, they will be instructed to increase intensity/volume if Borg is under that range or decrease it if Borg is above that range. (iii) The duration: the duration of each 
exercise will be increased every week, so the patient will increase walking time from 5 to 20 minutes, stepping up and down from 3 to 15 minutes and sitting and standing from 1 to 3 minutes (see progression in appendix 3 ). If a patient Do not possess a step (20-25 centimeters) the researchers will provide one during the period of the research. Additionally, patients will be instructed to walk at flat floor (may be in the backyard, hallway or on the street).

\section{Upper limbs strengthening}

The load adopted on upper limbs strengthening will vary from 50 to $100 \%$ of the verified load in the 10 maximum repetition test performed in the diagonal of the movement (see progression in appendix 3 ). In order to execute the exercises at home, patients will be instructed to use food packages and the load will be progressively increased every week. There will be also a variation in the number of sets (from two to four) and repetitions (from 10 to 15) (see progression in appendix 3 ). If they do not have the food packages to execute the exercise at home, the researchers will provide free weights.

\section{Stretching}

Stretching will be performed on the cervical region, upper and lower limbs, according to the model illustrated in the educational booklet (Appendix 2). Patients will be instructed to remain for twenty seconds in each position.

\section{Relaxation}

Patients will be seated in a relaxing position by five to ten minutes with relaxation music and calm breathing.

The information on the intensity, progression and duration of each prescribed step, as well as the activity performed at home, will be recorded in the individual monitoring form (Appendix 3).

\section{Randomized groups}

Instruction group (IG): Patients will have one physiotherapist-supervised session, which will be the first day of PRP. This group will receive in that session its personalized booklet which will be read alongside with the physiotherapist and all exercises will be explained and performed with the physiotherapist. On the next seven weeks of PRP, the patient will execute the exercises at home with equipment's that they have at home (chair, step, food packages) and they will be instructed to call for the physiotherapist if any doubt. In addition, patients will take a diary to register exercise habits during the week and they will be asked to return the table at the end of the eight-week period.

Patients will receive general exercise instructions to maintain a regular medication schedule and to do not execute the exercise after luch or dinner, or in the signs of infection, fever, dizziness or thoracic pain. If the patients have any experience of discomfort, they should look for health professional.

\section{Supervised group (SG)}


Patients will receive the same booklet of IG and, for a eight-week period, they will have a weekly supervised physiotherapy session (at laboratory) in where they will perform the same training they should do at home. All patients will be encouraged to exercise following the booklet at home more four times every week.

During the supervised sessions, patients will be monitored regarding pulse oximetry and Borg scale and values will be registered on a vital sign monitoring form (Appendix 4) and a clinical evolution form will be filled by physiotherapist.

At the end of PRP, patients of both groups will be submitted to a reassessment (the same executed prerehabilitation, except of spirometry) and the presence of $75 \%$ of the session will be considered a positive adherence.

The plans for communicating important protocol modifications will be registered at all to relevant parties (REBEC (trial registries, Ethics Committee of the Federal University of São Carlos investigators, trial participants, journals and financial support)

\section{Statistical analysis}

Data entry will be management by assessor blinded (not involved by rehabilitation) and the data will be registered in a documents clouds. All data will be analyzed by Statistical Package for the Social Sciences (SPSS) version 21.0. Anova Two Way Mixed Effect will be used to compare intergroup e intragroup (pre and post intervention) changes, considering significant a $p$ value $<0.05$. The sample size was calculated using the results of a pilot study with ten patients, and considering an effect size of 0.33 , an expected power of $80 \%$ and alpha-error $5 \%, 36$ patients will be needed and dropout was considered $10 \%$, totalized 40 patients. For the missing data, multiple imputation followed by a sensitivity analysis will be conducted.

The main researches will have access of the interim analyses and they will make the final decision to determinate the trial.

\section{Discussion}

Potential impact and significance of the study: It is expected that a low-cost once a week supervised rehabilitation program complemented with home exercises will present positive results especially on exercise capacity, which will make available a more excessive and effective PRP for patients with COPD.

Contribution and clinical applicability: The mains contributions will be a more accessible alternative rehabilitation protocol for patients with COPD patients, which will still have individualized prescription, hence, more effective than previous protocols. Therefore, accessibility is a key point for extend the rehabilitation opportunity for more COPD patients execute PRP an alternative place as home, basic health unit or community. We expected that the protocol could be adapted and applied in different moments, as 
the vacation of University Ambulatory or in different population, as liver transplantation, chronic kidney disease and others lungs diseases.

Strengths and limitations of the study: It is limitation the absent of lower limbs strengthening justified by the focus of the protocol is based on functional physical tests.

\section{Declarations}

Funding source: Regular Project funding by Fundação de Amparo à Pesquisa do Estado de São Paulo (FAPESP) Process FAPESP: $n^{\circ}$ 2018/06970-5 (appendix 5) and Coordenação de Aperfeiçoamento de Pessoal de Nível Superior (CAPES). The study is funded by FAPESP, and it will not have any role in study design, collection, management, analysis and interpretation of the data. Moreover, it will not have authority to change any aspect of the protocol or the writing manuscript.

The trials status: The trial was registered at URL: http://www.ensaiosclinicos.gov.br/rg/RBR-533hht/ with Register Number RBR-533hht. The date of registration and start data was September 20, 2018, the actual status is ongoing and the end prevision is December, 2020. The protocol was approved by Research Ethics Committee of the Federal University of São Carlos (UFSCar) number 4.348.948, CAAE: 85901318.0.0000.5504.

Competing interests: The authors declare no conflicting of interest.

Availability of data and materials: Not applicable.

Authors' contributions: MMCS, JFA, VAPDL was the responsible for elaborating and describe the protocol and MMCS is responsible for data collection and intervention collaboration. All authors read and approved the final manuscript.

Authors will be eligible when they have contributed to the design, collection or scientific writing of the research. The author not intend use of professional writers.

Ethics approval and consent to participate: The research ethics committee of UFSCAR approved the study ( number 4.348.948). All volunteers have been and will assigned an informed consent form.

Consent for publication: All volunteers have been and will be assigned to consent for publication.

Acknowledgements: Acknowledgements to Nathalia Maria Souza, Bruna Shara Vidal de Oliveira and Daiane Viana technical support of this research.

\section{References}

1. Zuniga Dourado V, Erico Tanni S, Alves Vale S, Maria Faganello M, Figueirôa Sanchez F, Godoy I. Manifestações sistêmicas na doença pulmonar obstrutiva crônica* Systemic manifestations in 
chronic obstructive pulmonary disease. J Bras Pneumol [Internet]. 2006;32(2):161-71. Available from: http://www.scielo.br/pdf/jbpneu/v32n2/a12v32n2.

2. Shrikrishna D, Hopkinson N. Chronic obstructive pulmonary disease: consequences beyond the lung. Clin Med (Northfield II) [Internet]. 2012;12(1):71-4. Available from: http://www.clinmed.rcpjournal.org/content/12/1/71.full.

3. da Costa JN, Arcuri JF, Goncalves IL, Davi SF, Pessoa BV, Jamami M, et al. Reproducibility of Cadence-Free 6-Minute Step Test in Subjects with COPD. Respir Care [Internet]. 2014;59(4):538-42. Available from: http://rc.rcjournal.com/cgi/doi/10.4187/respcare.02743.

4. Pradella CO, Belmonte GM, Maia MN, Delgado CS, Luise APT, Nascimento OA, et al. Home-Based Pulmonary Rehabilitation for Subjects With COPD: A Randomized Study. Respir Care [Internet]. 2015;60(4):526-32. Available from: http://rc.rcjournal.com/cgi/doi/10.4187/respcare.02994.

5. Camillo CA, Langer D, Osadnik CR, Pancini L, Demeyer H, Burtin C, et al. Survival after pulmonary rehabilitation in patients with COPD: impact of functional exercise capacity and its changes. 2016;2671-9.

6. Holland AE, Mahal A, Hill CJ, Lee AL, Burge AT, Cox NS, et al. Home-based rehabilitation for COPD using minimal resources: a randomised, controlled equivalence trial. Thorax [Internet]. 2017;72(1):57-65. Available from: http://thorax.bmj.com/lookup/doi/10.1136/thoraxjnl-2016208514.

7. Watz H, Pitta F, Rochester CL, Garcia-Aymerich J, ZuWallack R, Troosters T, et al. An official European respiratory society statement on physical activity in COPD. Eur Respir J. 2014;44(6):1521-37.

8. Tsai LLY, McNamara RJ, Moddel C, Alison JA, McKenzie DK, McKeough ZJ. Home-based telerehabilitation via real-time videoconferencing improves endurance exercise capacity in patients with COPD: The randomized controlled TeleR Study. Respirology. 2017;22(4):699-707.

9. Do Nascimento ESP, Sampaio LMM, Peixoto-Souza FS, Dias FD, Dantas Gomes ELF, Greiffo FR, et al. Home-based pulmonary rehabilitation improves clinical features and systemic inflammation in chronic obstructive pulmonary disease patients. Int J COPD. 2015;10:645-53.

10. Horton EJ, Mitchell KE, Johnson-Warrington V, Apps LD, Sewell L, Morgan M, et al. Comparison of a structured home-based rehabilitation programme with conventional supervised pulmonary rehabilitation: A randomised non-inferiority trial. Thorax. 2018;73(1):29-36.

11. Holland AE, Mahal A, Hill CJ, Lee AL, Burge AT, Moore R, et al. Benefits and costs of home-based pulmonary rehabilitation in chronic obstructive pulmonary disease - a multi-centre randomised controlled equivalence trial. BMC Pulm Med [Internet]. 2013;13(1):1. Available from: BMC Pulmonary Medicine.

12. Holland AE, Spruit MA, Troosters T, Puhan MA, Saey D, Mccormack MC, et al. An official European Respiratory Society / American Thoracic Society technical standard: field walking tests in chronic respiratory disease. Eur Respir J [Internet]. 2014;44(6):1428-46. Available from: https://www.thoracic.org/statements/resources/copd/FWT-Tech-Std.pdf.

13. Pereira CADC. Espirometria. J Bras Pneumol. 2002;28(supl 3):1-82. 
14. Dal Corso S, Duarte SR, Neder JA, Malaguti C, de Fuccio MB, de Castro Pereira CA, et al. A step test to assess exercise-related oxygen desaturation in interstitial lung disease. Eur Respir $\mathrm{J}$. 2007;29(2):330-6.

15. Pessoa BV, Arcuri JF, Labadessa IG, Costa JNF, Sentanin AC, Di Lorenzo V a P. Validity of the sixminute step test of free cadence in patients with chronic obstructive pulmonary disease. Brazilian $\mathrm{J}$ Phys Ther [Internet]. 2014;18(3):228-36. Available from: http://www.ncbi.nlm.nih.gov/pubmed/25003275.

16. Arcuri JF, Borghi-Silva A, Labadessa IG, Sentanin AC, Candolo C, Di Lorenzo VAP. Validity and reliability of the 6-minute step test in healthy individuals: A Cross-sectional study. Clin J Sport Med. 2016;26(1):69-75.

17. Iwama AM, Andrade GN, Shima P, Tanni SE, Godoy I, Dourado VZ. The six-minute walk test and body weight-walk distance product in healthy Brazilian subjects. Brazilian J Med Biol Res. 2009;42(11):1080-5.

18. Crook S, Büsching G, Schultz K, Lehbert N, Jelusic D, Keusch S, et al. A multicentre validation of the 1min sit-to-stand test in patients with COPD. Eur Respir J [Internet]. 2017;49(3):1-11. Available from: http://dx.doi.org/10.1183/13993003.01871-2016.

19. Strassmann A, Steurer-Stey C, Lana KD, Zoller M, Turk AJ, Suter P, et al. Population-based reference values for the 1-min sit-to-stand test. Int J Public Health. 2013;58(6):949-53.

20. Mahler DA, Wells CK. Evaluation of clinical methods for rating dyspnea. Chest. 1988;93(3):580-6.

21. POLLOCK ML; WILMORE JHE. Exercícios na saúde e na doença: De, avaliação e prescrição para a prevenção e reabilitação. 2ª . LTDA editora M e C, editor. Rio Janeiro; 1996.

22. Jones PW, Harding G, Berry P, Wiklund I, Leidy NK. Development and first validation of the COPD Assessment Test. Eur Respir J. 2009;34(3):648-54.

23. Godfrey A, Culhane KM, Lyons GM. Comparison of the performance of the activPAL ${ }^{\mathrm{TM}}$ Professional physical activity logger to a discrete accelerometer-based activity monitor. Med Eng Phys. 2007;29(8):930-4.

24. Schneider LP, Furlanetto KC, Rodrigues A, Lopes JR, Hernandes NA, Pitta F. Sedentary Behaviour and Physical Inactivity in Patients with Chronic Obstructive Pulmonary Disease: Two Sides of the Same Coin? COPD J Chronic Obstr Pulm Dis [Internet]. 2018;15(5):432-8. Available from: https://doi.org/10.1080/15412555.2018.1548587.

\section{Figures}




\begin{tabular}{|c|c|c|c|c|}
\hline & \multicolumn{4}{|c|}{ Study period } \\
\hline & Enrolment & Evaluation & Intervention & Re-evaluation \\
\hline TIMEPOINT & $-t_{1}$ & $t_{0}$ (weesk 0-1) & $\begin{array}{c}t_{l} \\
\text { (woekk 1-8) }\end{array}$ & $T_{2}$ (week $9=10$ ) \\
\hline \multicolumn{5}{|l|}{ ENROLMENT: } \\
\hline \multirow{3}{*}{$\begin{array}{r}\text { Informed consent } \\
\text { Randomization and } \\
\text { Allocation }\end{array}$} & $\mathrm{x}$ & & & \\
\hline & $\mathrm{x}$ & & & \\
\hline & & $\mathrm{x}$ & & \\
\hline \multicolumn{5}{|l|}{ INTERVENTIONS: } \\
\hline Instruction group & & & $\mathrm{x}$ & \\
\hline Supervised group & & & $\mathrm{x}$ & \\
\hline \multicolumn{5}{|l|}{ ASSESSMENTS: } \\
\hline $\begin{array}{r}\text { Baseline } \\
\text { characteristcs* }\end{array}$ & & $\mathrm{x}$ & & \\
\hline $\begin{array}{r}\text { Exercise capacity } \\
\text { tests** }\end{array}$ & & $\mathrm{x}$ & & $\mathrm{x}$ \\
\hline $\begin{array}{r}\text { Dyspnea } \\
\text { sensation**** }\end{array}$ & & $\mathrm{x}$ & & $\mathrm{x}$ \\
\hline $\begin{array}{l}\text { Upper limb muscle } \\
\text { strength } * * *\end{array}$ & & $\mathrm{x}$ & & $\mathrm{x}$ \\
\hline Quality of life $* * * * *$ & & $\mathrm{x}$ & & $\mathrm{x}$ \\
\hline $\begin{array}{l}\text { Physical Activity } \\
\text { daily living }\end{array}$ & & $\mathrm{x}$ & & $\mathrm{x}$ \\
\hline
\end{tabular}

*Baseline characteristics include pulmonary function assessed by spirometry, information about the patients' anthropometric data, schooling level, presence of continuous use of oxygen therap' active smoking and comorbidities (Charlson Comorbidity Index).

** *Exercise capacity test includes Six minute step test, six minute walk test and one minute sit to stand test

*** Dyspnea sensation includes Modified Medical Research Council (mMRC).

**** Upper limb muscle strength includes Ten repetition maximum test.

*****Quality of life includes The Saint George Respiratory Questionnaire (SQRQ).

******Physical Activity daily living (PADL) includes actigraph activPAL3TM.

\section{Figure 1}

Standard Protocol Items: Recommendations for Interventional Trials (SPIRIT) checklist providing information about volunteer recruitment and variables evaluated in each period according to SPIRIT recommendations. 


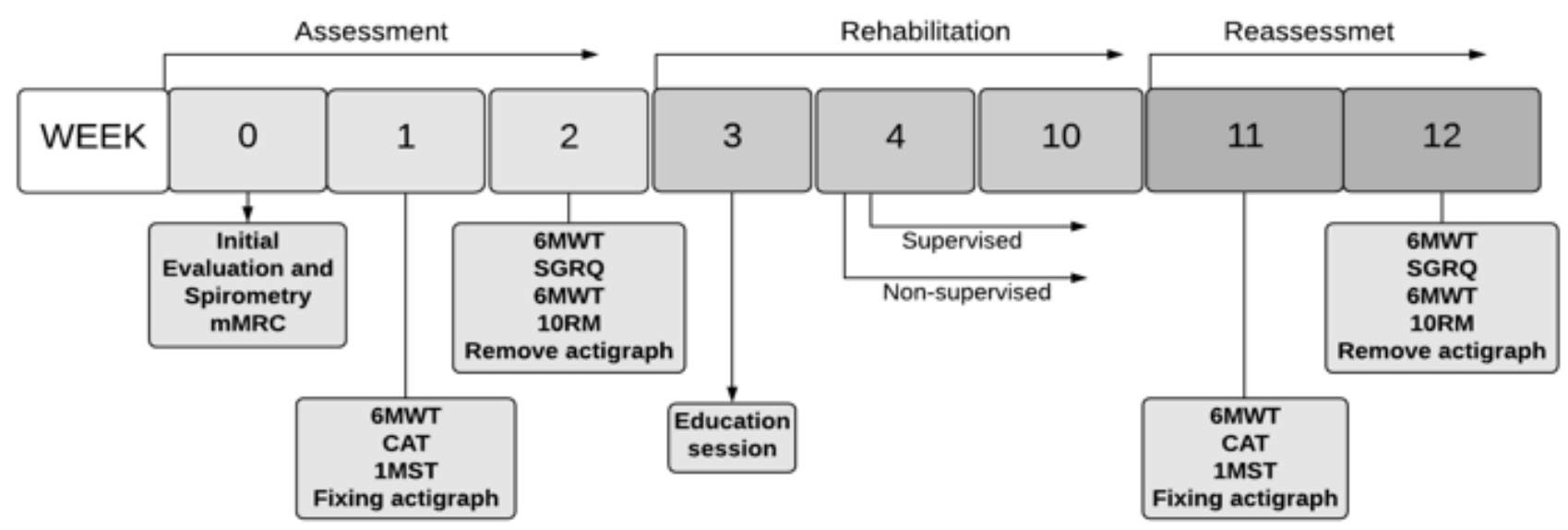

mMRC: Modified Medical Research Council ; 6MWT: Six minute walk test; IMST: One minute sit to stand test; 6MST: six-minute step test ; 10RM: Ten repetition maximum test; SGRQ: Saint George Respiratory Questionnaire; CAT: COPD Assessment Test.

Figure 2

Experimental design of study

\section{Supplementary Files}

This is a list of supplementary files associated with this preprint. Click to download.

- Appendix.docx

- SPIRITFillablechecklist15Aug2013.doc 